Psychometric Network Analysis of the Hungarian WAIS

Christopher J. Schmank ${ }^{1}$, Sara Anne Goring ${ }^{1}$, Kristof Kovacs ${ }^{2}$, \& Andrew R. A. Conway ${ }^{1}$

Claremont Graduate University ${ }^{1}$

ELTE Eotvos Lorand University ${ }^{2}$ 


\begin{abstract}
The positive manifold - the finding that cognitive ability measures demonstrate positive correlations with one another-has led to models of intelligence that include a general cognitive ability or general intelligence $(g)$. This view has been reinforced using factor analysis and latent variable models. However, a new theory of intelligence, Process Overlap Theory (POT; Kovacs \& Conway, 2016), posits that $g$ is not a psychological attribute but an index of cognitive abilities that results from an interconnected network of cognitive processes. From this perspective, psychometric network analysis is an attractive alternative to latent variable modeling. Network analyses display partial correlations among observed variables that demonstrate direct relationships among observed variables. To demonstrate the benefits of this approach, the Hungarian Wechsler Adult Intelligence Scale Fourth Edition (H-WAIS-IV; Wechsler, 2008) was analyzed using both psychometric network analysis and latent variable modeling. Network models were directly compared to latent variable models. Results indicate that the H-WAIS-IV data was better fit by network models than by latent variable models. We argue that POT, and network models, provide a more accurate view of the structure of intelligence than traditional approaches.
\end{abstract}




\section{Psychometric Network Analysis of the Hungarian WAIS}

One of the most replicated findings in psychological science is the positive manifold. The positive manifold refers to the finding that all cognitive ability measures tend to be positively correlated. Individuals who score above average on one test of cognitive ability (e.g., verbal reasoning) tend to score above average on other tests of cognitive ability (e.g., numerical reasoning). Beginning with Spearman (1904), the positive manifold has been explained by submitting test scores to factor analysis and extracting a single common factor, $g$. The most common interpretation of $g$ is that it reflects a general cognitive ability that is important across a range of different tasks and extremely variable across people. General cognitive ability is an intra-individual psychological attribute that explains inter-individual differences in cognitive performance. Indeed, this view of intelligence has been widely accepted in psychological science (Gottfredson, 1997; Reeve \& Charles, 2008).

However, a new approach, Process Overlap Theory (POT; Kovacs \& Conway, 2016), challenges the conventional view of intelligence, specifically the interpretation of $g$ as general cognitive ability. According to POT, the positive manifold does not reflect a general cognitive ability. In fact, according to POT, there is no such thing as a general cognitive ability. Instead, the observed covariance in tests of intelligence is due to overlapping sets of cognitive processes required to perform the tests. The cognitive processes required to perform different tests may be domain-general or they may be domain-specific. During cognitive task performance the larger the extent that domain-general or executive functioning processes overlap with domain-specific processes the larger the correlation between different tests. Therefore, according to POT, there is not a single common cause of variance in tests; there are multiple causes, and some are more general while some are more specific (Kovacs \& Conway, 2016). 
In the intelligence literature the most accepted models are reflective higher-order models. In such models there are levels of ability, the higher the level the broader the ability with a general factor, $g$, on the top of the hierarchy. This means that tests reflect the same general factor to some extent, and usually this superordinate factor is interpreted as general cognitive ability. POT, is incompatible with such latent variable models of intelligence. Instead, POT proposes that $g$ is a formative construct, i.e., the common consequence rather than the common cause of the correlations between tests. Therefore, $g$ does not explain the positive manifold, the overlap between processes does. Moreover, latent variables in factor models are not process pure and the same processes are tapped by several factors (hence the overlap). Therefore, from a cognitive perspective that aims at understanding intelligence at the process level a psychometric network modeling approach is more appropriate than a latent variable modeling approach.

In the current study we compare latent variable models of intelligence to psychometric network models of intelligence, using data from the Hungarian Wechsler Adult Intelligence Scale Fourth Edition (H-WAIS-IV; Wechsler, 2008). First, a traditional example of latent variable modeling is presented, and key advantages and disadvantages of latent variable models are considered. Next, psychometric network analysis is presented as an alternative to latent variable modeling. This is followed by a discussion of a recent publication that directly compares a psychometric network model to a nested latent variable model (Kan, van der Maas, \& Levine, 2019). Finally, the psychometric network model and latent variable model of H-WAIS-IV data are presented and directly compared using model fit indices.

\section{Latent Variable Models}

Latent variable modeling allows researchers to explain the covariation among many observed variables and explore the underlying structure of data using multiple unobserved 
variables (Gorsuch, 1983; Kline, 2005; Yong \& Pearce, 2013). In fact, it was Spearman’s original use of factor analysis in 1904 that began and led to the overall acceptance of modeling intelligence using hierarchical and reflective latent variable models.

Advantages of Latent Variable Models. One advantage of latent variable modeling is that the technique allows for both determination and confirmation of the underlying structure of covariation among observed variables. A second advantage of latent variable modeling is that the technique reduces large datasets into fewer and more easily interpretable numbers of unobserved latent factors (Field, Miles, \& Field, 2012). For instance, McGrew (2011) conducted latent variable modeling on the Wechsler Adult Intelligence Scale Third Edition that consisted of 15 subtests. Following latent variable modelling, 5 latent factors were extracted that sufficiently explained the covariation among the original 15 observations, achieving data reduction by $67 \%$ while simultaneously establishing the underlying latent structure of intelligence data. A third advantage of latent variable modeling is that the technique allows for the improvement of questionnaires or cognitive test batteries used to measure unobserved, latent concepts or factors. By using previously established latent variable models or theoretically motivated latent factor structures, items can be assessed for how well they map onto these unobserved factors. In summary, the use of latent variable modeling attempts to explain as many observed items by as few unobserved latent concepts as possible. Although, latent variable modeling has well established advantages, several substantively relevant disadvantages exist and are discussed next.

Disadvantages of Latent Variable Models. There are two major disadvantages of latent variable modeling. First, there is often a lack of consensus on how latent variables should be defined and interpreted (Bollen, 2002; Borsboom, Mellenbergh, \& van Heerden, 2003; Conway \& Kovacs, 2013). Definitions of latent variables tend to be subjective; leaving the researcher 
with the final say on what specific latent variables represent (for more information on this limitation see Bock, Goode, \& Webb, 2003). Additionally, a posteriori or data driven latent variables might be sample dependent and not truly representative of some specific psychological attribute (Bollen, 2002). Consider the Cattell-Horn-Carroll (CHC) model of intelligence (Figure 1; Carroll, 1993; Cattell, 1943; Horn \& Cattell, 1966). Hierarchical (i.e., higher-order) models like the CHC, theoretically imply that scores on observed variables are directly influenced by ability-specific cognitive processes that are each in turn influenced by general cognitive ability. However, this is solely an interpretation of these latent variables based on historical and theoretical precedence and may not be truly representative of human intelligence.

A second disadvantage of latent variable models is the principle of local independence. Latent variables are considered the common cause of related observations, so any covariance among observations is fully explained by the latent variable. In a latent variable model, this implies that the observations are locally independent from one another (Borsboom et al., 2003). For example, observations of cognitive ability like vocabulary, reading comprehension, and listening comprehension would be explained by a single latent variable called verbal ability. The principle of local independence implies that verbal ability accounts for all the shared variance among these cognitive tasks and that no other relationships or shared variance exists among these observations; these variables are ostensibly independent (for more information regarding comorbidity and latent variable models see Cramer, Waldorp, van der Maas, \& Borsboom, 2010; McNally, 2016). The consequence here is that once manifest variables are explained by a latent variable they pose no direct nor indirect effects on one another.

Interim Summary. Historically, latent variable modeling has been widely applied in psychological research. However, due to the subjective nature of the interpretation of extracted 
latent variables, researchers cannot be sure whether these latent variables directly map onto real psychological attributes or if they should be considered psychometric artifacts. Recently, researchers have begun to pursue topics traditionally explored using latent variable modeling from a different perspective called psychometric network analysis, e.g., depression (Madhoo \& Levine, 2016; van Borkulo et al., 2015) and post-traumatic stress disorder (Fried et al., 2018; McNally et al., 2015).

\section{Psychometric Network Analysis}

Researchers using psychometric network analysis assess observed variables and the estimated partial correlations among these observations, without assuming latent common causes. In latent variable modeling, unobserved latent factors are estimated from the variancecovariance patterns among observed variables. On the other hand, psychometric network analysis conceptualizes complex psychological attributes or behaviors as interconnected networks. In terms of network science and network modeling, observations are referred to as nodes and the connections between pairs of nodes are referred to as edges. More recently, Kan, et al. (2019) provided a description of the differences between traditional latent variable modeling and psychometric network analysis. Kan et al. proposed that psychometric network analysis lends itself to theories of intelligence like POT (Kovacs \& Conway, 2016) and their own model of intelligence known as mutualism (van der Maas et al., 2006) because these theoretical models of intelligence imply that cognitive processes and abilities interact dynamically with one another. Additionally, Kan et al. (2019) provided evidence that compared to latent variable models, psychometric network models fit intelligence data better.

Using modified R code originally provided by Kan et al. (2019) and van der Maas et al. (2017), as well as guidelines established by Epskamp and Fried (2018) we assessed the overall fit 
of H-WAIS-IV data using both latent variable modeling (e.g., higher-order $g$ model) and psychometric network analysis. First, confirmatory factor analyses were conducted using the CHC higher-order model of intelligence as a measurement model. Next, psychometric network analyses were conducted. Finally, the model fit indices extracted from confirmatory factor analysis and psychometric network models of H-WAIS-IV data were directly compared.

\section{Method}

\section{Subjects}

Sample consisted of 1,112 people between 12 and 90 years of age $(M=45.15 ; S D=$ $22.85 ; N=1,110 ; 646$ women). Subjects' reported level of education indicated that $36.60 \%$ had completed primary school, $23.47 \%$ completed some vocational training, $14.30 \%$ completed a college or university degree, $12.95 \%$ completed grammar school (one version of high school in Hungary), $10.43 \%$ completed vocational school (another version of high school in Hungary), and $2.25 \%$ failed to complete primary school. The sample is representative of the population of Hungary in terms of age, geographical location, type of settlement, education, and gender according to the latest census conducted before the standardization of the WAIS.

\section{Measures}

The H-WAIS-IV consisted of 15 subtests: Information, Vocabulary, Comparisons, Similarities, Picture Completion, Block Design, Figure Weights, Matrix Reasoning, Visual Puzzles, Arithmetic, Digit Span, Letter-Number Sequencing, Cancellation, Coding, and Symbol Search. Information about these measures can be found in the Technical and Interpretative Manual (Wechsler, 2008).

\section{Statistical Procedure}

\section{Model Fit Evaluation for Latent Variable and Psychometric Network Models. A} general approach to model fit evaluation was followed as provided by Kline (2005) to assess 
both psychometric network models and latent variable models. Additionally, due to the continuous nature of the H-WAIS-IV data, model fit evaluation was conducted using suggestions by $\mathrm{Hu}$ and Bentler (1999) and cutoff criteria published by Schreiber, Stage, King, Nora, and Barlow (2006). Thus, model fit will be deemed appropriate when (a) the ratio of model chisquare $\left(\chi^{2}\right)$ to degrees of freedom is less than or equal to 3.00, (b) comparative fit indices (e.g., Comparative Fit Index $[\mathrm{CFI}]$ and Tucker-Lewis Index [TLI]) greater than or equal to .95, and (c) Root Mean Square Error of Approximation (RMSEA) values less than or equal to .06. Additionally, Akaike Information Criteria (AIC) and Bayesian Information Criteria (BIC) values were compared; smaller values indicated better fit for each fit index. Finally, $\chi^{2}$ difference tests were employed to directly compare the nested psychometric network model to the latent variable model.

Confirmatory Factor Analysis. Factor analyses were conducted to assess the model fit of the H-WAIS-IV assuming the CHC higher-order model of intelligence. Assuming this model implies six latent variables hierarchically arranged with one superordinate second-order latent variable representing $g$, and five subordinate first-order latent variables representing crystallized intelligence $\left(G_{\mathrm{c}}\right)$, fluid reasoning $\left(G_{\mathrm{f}}\right)$, visuospatial ability $\left(G_{\mathrm{v}}\right)$, working memory $\left(G_{\mathrm{wm}}\right)$, and processing speed $\left(G_{\mathrm{s}}\right)$. Measures associated with crystallized intelligence demonstrate excellent test-retest reliability $\left(r_{G \mathrm{c}}=.81-.93\right)$. Measures of fluid reasoning, visuospatial ability, working memory, and processing speed all demonstrated medium to high test-retest reliabilities $\left(r_{G \mathrm{f}}=\right.$ $.70-.85 ; r_{G \mathrm{v}}=.57-.81 r_{G \mathrm{wm}}=.70-.89 ; r_{G \mathrm{~s}}=.67-.86$; c.f., Sattler \& Ryan, 2009, p. 38). Latent variable models were conducted using the lavaan (Rosseel, 2012) and openMx (Neale et al., 2016) packages in R (R Core Team, 2013) and were visualized using Onyx (van Oertzen, Brandmaier, \& Tsang, 2015). 
Psychometric Network Analysis. Following the latent variable analyses, psychometric network analyses were conducted on correlation matrices extracted from the H-WAIS-IV data. Psychometric network analysis was conducted using the qgraph (Epskamp, Cramer, Waldorp, Schmittmann \& Borsboom, 2012) and openMx (Neale et al., 2016) packages in R and were visualized using qgraph. Guidelines provided by Epskamp and Fried (2018) were followed when conducting psychometric network analysis. Network models were generated using the graphical least absolute shrinkage and selector operator (gLASSO) regularization method to control network sparsity (Friedman, Hastie, \& Tibshirani, 2008). Using the gLASSO regularization technique involves manually setting two parameters: (a) the hyperparameter gamma $(\gamma)$ and (b) the tuning parameter lambda $(\lambda)$. In following the tutorial provided by Epskamp and Fried (2018) the hyperparameter was set conservatively $(\gamma=.50)$. Setting $\gamma$ conservatively reflects the extended BIC gLASSO regularization technique that prefers simpler models with fewer estimated edges. Additionally, in following Epskamp, Lunansky, Tio, and Borsboom (2018, April [Blog post]) the tuning parameter was set modestly $(\lambda=.01)$ reflecting a technique that limits spurious edges while retaining as many true edges as possible.

Following the guidelines of Epskamp and Fried (2018) and setting the parameters in this manner allowed resulting psychometric network models to be estimated with high specificity, as is typical of gLASSO regularization, as well as high sensitivity, due to reduction of false-positive edges that is typical when setting the tuning parameter low. The gLASSO regularization technique was followed to ensure the removal of estimated edges that were spurious (i.e., falsepositive) or only occurring due to sampling error. This technique was only available for the psychometric network analyses conducted in qgraph as there currently is no way to specify these parameters using openMX. 


\section{Results}

\section{Data Preparation}

Several variables were missing data in the H-WAIS-IV (Wechsler, 2008) dataset; missing data were imputed via multivariate imputation techniques provided by the mice (van Buuren $\&$ Groothuis-Oudshoorn, 2011) and VIM (Kowarik \& Templ, 2016) packages in $\mathrm{R}^{1}$.

\section{Correlations, Descriptive Statistics, and Reliability}

The correlation matrix for H-WAIS-IV data is presented in Table 1, with means and standard deviations presented in the bottom two rows. The H-WAIS-IV data demonstrated statistical reliability (Cronbach, 1951; Nunnally, 1978; Nunnally \& Bernstein, 1994), with overall excellent internal consistency (Cronbach's $\alpha=.94,95 \%$ CI $[.93, .95]$ ). Additionally, the internal consistency across the cognitive constructs present in $\mathrm{CHC}$ models of intelligence indicated excellent reliability for the construct representing crystallized intelligence $\left(G_{\mathrm{c}} ; \alpha=.91\right.$, $95 \%$ CI $[.90, .92])$; good reliability for the constructs representing fluid reasoning $\left(G_{\mathrm{f}} ; \alpha=.84\right.$, $95 \%$ CI $[.83, .86])$, visuospatial ability $\left(G_{\mathrm{v}} ; \alpha=.82,95 \%\right.$ CI $\left.[.80, .84]\right)$ and working memory $\left(G_{\mathrm{wm}} ; \alpha=.82,95 \% \mathrm{CI}[.80, .84]\right)$; and acceptable reliability for the construct representing processing speed $\left(G_{\mathrm{s}} ; \alpha=.79,95 \%\right.$ CI $\left.[.77, .82]\right)$.

\section{Confirmatory Factor Analysis and Psychometric Network Models of the H-WAIS-IV}

Model fit indices for the confirmatory factor analyses and the psychometric network analyses are presented in Table 2. On the one hand, the latent variable models demonstrated varied fit across all fit indices reported. First, these latent variable models demonstrated unacceptable $\chi^{2}$ values and problematic values for the ratio between $\chi^{2}$ and degrees of freedom.

\footnotetext{
${ }^{1}$ Missing data was present for more than $15 \%$ of the sample for the following cognitive ability measures: (a) LetterNumber Sequencing, (b) Figure Weights, and (c) Cancellation tasks. Prior to implementing the imputation techniques, the sample size was 875 . Following multivariate imputation techniques the sample size increased to 1,112 subjects.
} 
Second, these latent variable models demonstrated values in the acceptable range for comparative fit indices (i.e., CFI and TLI) and RMSEA. For a visualization of the H-WAIS-IV data fit to this latent variable structure see Figure 2. This figure can be interpreted in the following manner: (a) starting at the bottom each H-WAIS-IV item has a uniqueness value where larger values reflect greater unaccounted variance; (b) each first-order latent variable accounts for some degree of variance in items that is represented by the directional arrows connecting latent variables $\left(G_{\mathrm{c}}, G_{\mathrm{f}}, G_{\mathrm{v}} G_{\mathrm{wm}}\right.$, and $\left.G_{\mathrm{s}}\right)$ to items (i.e., represented by boxes); and (c) the general cognitive ability factor loadings onto each first-order latent variable is represented by the direction arrows connecting the superordinate $g$ factor to first-order latent variables.

On the other hand, unlike the latent variable models, the psychometric network models demonstrated excellent fit across most fit indices reported. First, the psychometric network model generated using qgraph demonstrated statistically non-significant $\chi^{2}$ values and values of the ratio between $\chi^{2}$ and degrees of freedom well below the value deemed acceptable above. The psychometric network model generated using openMX demonstrated a statistically significant $\chi^{2}$ values, however the value of the ratio between $\chi^{2}$ and degrees of freedom was well below the acceptable value. Second, the comparative fit indices (i.e., CFI and TLI) both demonstrated perfect fit and RMSEA was smaller compared to the latent variable models. Further evidence of the superiority of the psychometric network models was evidenced by the smallest AIC and BIC values across Table 2. For the visualization of these psychometric network models see Figure 3 and Figure 4. Figure 3 represents a weighted, undirected network model of the H-WAIS-IV data and Figure 4 represents a weighted, directed network model of H-WAIS-IV data. For simplicity, nodes have been colored to reflect the latent structure of the estimated latent variable models and the width of each line the amount of association between pairs of nodes. The network models 
reveal 4 to 5 clusters of nodes. Three of the clusters are distinct, representing working memory, processing speed, and crystallized intelligence. The distinction between fluid reasoning and visuospatial ability is less clear. Also, in both network models, working memory and fluid reasoning are more central to the network than the other clusters.

Finally, $\chi^{2}$ difference tests were conducted to directly compare the psychometric network models and to directly compare each network model to the latent variable model conducted using the same statistical package. First, the $\chi^{2}$ difference test comparing the directed and undirected network models indicated that the undirected network model fit the data better, $\chi^{2}(12, N=1,112)$ $=38.76, p<.001$. Additionally, each subsequent $\chi^{2}$ difference test demonstrated that the psychometric network models generated using qgraph and openMx fit the data better than either of the latent variable models using lavaan and openMx, $\chi^{2}(37, N=1,112)=327.88, p<.001$ and $\chi^{2}(25, N=1,112)=289.11, p<.001$, respectively. First, this suggests that psychometric network models fit the H-WAIS-IV data better than the CHC higher-order latent variable models regardless of the statistical package used. Additionally, it demonstrates that the psychometric network analysis generated by qgraph fit the H-WAIS-IV data better than the network model generated by openMx.

\section{Discussion}

The purpose of this study was to consider psychometric network analysis as an alternative approach to latent variable modeling to investigate the structure of intelligence. To this end, psychometric network analysis and latent variable modeling was applied to H-WAIS-IV data (Wechsler, 2008). Direct comparisons of the estimated network models and confirmatory factor analyses indicated that psychometric network models fit intelligence data more accurately, and this is true regardless of the statistical package used (e.g., lavaan, qgraph, and openMx). The 
longstanding tradition in the psychological sciences of using structural equation modeling and other latent variable or factor analytic techniques is primarily due to the relative ease of using these statistical techniques. Additionally, these statistical procedures provide a useful technique for data reduction of complex data into fewer and arguably easier to comprehend factors or latent observations. However, when we applied latent variable analyses to the H-WAIS-IV dataset, the model fit indices were inconsistent across both latent variable models based on criteria provided by Schreiber et al. (2006). The present findings were consistent with findings presented by Kan et al. (2019), who demonstrated that the underlying structure of intelligence was better represented as a psychometric network and not a reflective latent variable model. Psychometric network models fit H-WAIS-IV intelligence data better than latent variable models. This supports theoretical models of intelligence like POT (Kovacs \& Conway, 2016) that theorize $g$ as a consequence of the interconnections among overlapping cognitive processes rather than a direct cause of these overlapping processes.

Many have criticized latent variable models of cognitive processes and intelligence because the observed variables tend not to be process pure. Process purity is violated when cognitive tasks are multifaceted and not representative of a single process. Consider the measure of symbol search from the H-WAIS-IV. While completing this task participants are provided with a target symbol that must be kept consciously activated while simultaneously conducting a thorough search of an array of non-target symbols. The process of keeping the target symbol active involves working memory while the search process involves processing speed. Thus, many cognitive tasks involve representations of multiple latent variables that increase the error and reduce the model fit of latent variable models of intelligence. 
Overall, when observed variables are not process pure (e.g., measures of various psychological attributes) problems with latent variable models follow. Unequivocally, the ability to estimate interconnections among observed variables in psychometric network models lends itself to statistical modeling techniques used on variables that are not process pure. These psychometric network modeling techniques can be used as an exploratory analysis to determine variables that cluster together. Like exploratory factor analysis, this could be a precursor to using more confirmatory techniques like confirmatory factor analysis. In fact, sometimes latent variable models do not fit data as well as theorized or hypothesized. In cases like this, network modeling can be used to visualize and assess the one-to-one relationships among observed variables that might illuminate reasons for poor model fit (Rhemtulla, van Bork, Epskamp, \& Isvoranu, 2018, May).

An additional suggestion is to implement the guidelines provided by Epskamp, Borsboom, and Fried (2018) regarding psychometric network stability analyses. This procedure employs bootstrapping techniques on full datasets to assess the overall accuracy and invariance of network models. This technique allows researchers to establish confidence intervals on an edge-by-edge basis. These confidence intervals can be used to determine the accuracy of each estimated edge in the network. Edges are estimated accurately when the confidence intervals surrounding them are relatively small. However, it should be noted that these confidence intervals do not function as an assessment of whether the edge weight significantly differs from zero. Additionally, due to the nature of confidence intervals edges can be directly compared using a nonparametric difference test to determine whether an edge in the network demonstrates statistically significant differences from other estimated edges. Thus, stability analyses could be used to elucidate whether network models are generalizable and invariant across datasets. 
In conclusion, psychometric network analyses fit H-WAIS-IV data better than the higherorder CHC latent variable model of intelligence across all model fit indices. Much like the work published by Kan et al. (2019) the better fit of psychometric network analyses to intelligence data supports theories of intelligence like POT (Kovacs \& Conway, 2016), that view $g$ as an index that emerges due to interconnections among cognitive tasks that sample many overlapping cognitive processes. The advantages of psychometric network modeling suggest that the psychological sciences should use this approach more often, whether researchers plan to employ these analyses prior to, without, or in concert with latent variable modeling. Additionally, researchers employing latent variable modeling techniques ought to consider the questions proposed by Borsboom et al. (2003) concerning latent variables:

Should we assume that the latent variable signifies a real entity or conceive of it as a useful fiction, constructed by the human mind? Should we say that we measure a latent variable in the sense that it underlies and determines our observations, or is it more appropriately considered to be constructed out of the observed scores? What exactly constitutes the relation between latent variables and observed scores? Is this relation of a causal nature? If so, in what sense? And, most important, is latent variable theory neutral with respect to these issues? (pp. 204)

Additional analyses of large-scale data sets applying both psychometric network modeling and latent variable models might further reveal the advantages of each approach. Moreover, a recent development, latent variable network modeling (Epskamp, Rhemtulla, \& Borsboom, 2017) combines the two approaches by describing a network of connections between latent variables that account for performance of tests of cognitive abilities. This might reconcile the two 
approaches, bringing the "best of both worlds" to research on the structure of human cognitive abilities. 


\section{References}

Bock, G. R., Goode, J. A., \& Webb, K. (2003). The Nature of Intelligence. Wiley.

Bollen, K. A. (2002). Latent variables in psychology and the social sciences. Annual Review of Psychology, 53, 605-34. https://doi.org/10.1146/annurev.psych.53.100901.135239

Borsboom, D., Mellenbergh, G. J., \& van Heerden, J. (2003). The theoretical status of latent variables. Psychological Review, 110, 203-219. https://doi.org/10.1037/0033295X.110.2.203

Carroll, J. B. (1993). Human Cognitive Abilities: A Survey of Factor-Analytic Studies. New York, NY: Cambridge University Press. https://doi.org/10.1017/CBO9780511571312

Cattell, R. B. (1943). The measurement of adult intelligence. Psychological Bulletin, 40, 153193. https://doi.org/10.1037/h0059973

Conway, A. R. A., \& Kovacs, K. (2013). Individual differences in intelligence and working memory: A review of latent variable models. In B. H. Ross (Ed.), The psychology of learning and motivation (pp. 233-270). San Diego, CA: Elsevier Academic

Press. https://doi.org/10.1016/B978-0-12-407237-4.00007-4

Cramer, A. O. J., Waldorp, L. J., van der Maas, H. L. J., \& Borsboom, D. (2010). Comorbidity: A network perspective. Behavioral and Brain Sciences, 33, 137-193.

https://doi.org/10.1017/S0140525X09991567

Cronbach, L. J. (1951). Coefficient alpha and the internal structure of tests. Psychometrika, 16, 297-334. https://doi.org/10.1007/BF02310555

Epskamp, S., Borsboom, D., \& Fried, E. I. (2018). Estimating psychological networks and their accuracy: A tutorial paper. Behavior Research Methods, 50, 195-212. https://doi.org/10.3758/s13428-017-0862-1 
Epskamp, S., Cramer, A. O. J., Waldorp, L. J., Schmittmann, V. D., \& Borsboom, D. (2012). qgraph: Network visualizations of relationships in psychometric data. Journal of Statistical Software, 48, 1-18. https://doi.org/10.18637/jss.v048.i04

Epskamp, S., \& Fried, E. I. (2018). A tutorial on regularized partial correlation networks. Psychological Methods, 23, 617-634. https://doi.org/10.1037/met0000167

Epskamp, S., Lunansky, G., Tio, P., \& Borsboom, D. (2018, April). Recent developments on the performance of graphical LASSO networks [Blog post]. Retrieved from http://psychosystems.org/author/sachaepskamp/

Epskamp, S., Rhemtulla, M., Borsboom, D. (2017). Generalized network psychometrics: Combining network and latent variable models. Psychometrika, 82, 904-927. https://doi.org/10.1007/s11336-017-9557-x

Field, A., Miles, J., \& Field, Z. (2012). Discovering Statistics Using R. Los Angeles, CA: SAGE Publications Inc.

Fried, E. I., Eidhof, M. B., Palic, S., Costantini, G., Huisman-van Dijk, H. M., . . Karstoft, K.-I. (2018). Replicability and generalizability of Posttraumatic Stress Disorder (PTSD) networks: A cross-cultural multisite study of PTSD symptoms in four trauma patient samples. Clinical Psychological Science, 6, 335-351. https://doi.org/10.1177/2167702617745092

Friedman, J., Hastie, T., \& Tibshirani, R. (2008). Sparse inverse covariance estimation with the graphical lasso. Biostatistics, 9, 432-441. https://doi.org/10.1093/biostatistics/kxm045

Gorsuch, R. L. (1983). Factor Analysis (2nd ed.). Hillsdale: NJ: Lawrence Erlbaum Associates. Gottfredson, L. S. (1997). Why g matters: The complexity of everyday life. Intelligence, 24, 79132. https://doi.org/10.1016/S0160-2896(97)90014-3 
Horn, J. L., \& Cattell, R. B. (1966). Refinement and test of the theory of fluid and crystallized general intelligences. Journal of Educational Psychology, 57, 253-270. https://doi.org/10.1037/h0023816

Hu, L. T., \& Bentler, P. M. (1999). Cutoff criteria for fit indexes in covariance structure analysis: Conventional criteria versus new alternatives. Structural Equation Modeling, 6, 1-55. https://doi.org/10.1080/10705519909540118

Kline, R. B. (2005). Principles and Practice of Structural Equation Modeling (4th ed.). New York, NY, USA: Guilford Press.

Kovacs, K., \& Conway, A. R. A. (2016). Process Overlap Theory: A unified account of the general factor of intelligence. Psychological Inquiry, 27, 151-177. https://doi.org/ 10.1109/WI-IAT.2010.197

Kowarik, A., \& Templ, M. (2016). Imputation with the R package VIM. Journal of Statistical Software, 74, 1-16. https://doi.org/10.18637/jss.v074.i07

Madhoo, M., \& Levine, S. Z. (2016). Network analysis of the quick inventory of depressive symptomology: Reanalysis of the STAR*D clinical trial. European Neuropsychopharmacology, 26, 1768-1774. https://doi.org/10.1016/j.euroneuro.2016.09.368

McGrew, K. S. (2011). Independent CFA of the French WAIS-IV. Retrieved from http://www.iqscorner.com/2011/04/ap101-brief-8-guest-brief-independent.html

McNally, R. J. (2016). Can network analysis transform psychopathology? Behaviour Research and Therapy, 86, 95-104. https://doi.org/10.1016/j.brat.2016.06.006

McNally, R. J., Robinaugh, D. J., Wu, G. W. Y., Wang, L., Deserno, M., \& Borsboom, D. (2015). Mental disorders as causal systems: A network approach to post-traumatic stress 
disorder. Clinical Psychological Science, 3, 836-849.

https://doi.org/10.1177/2167702614553230

Neale, M. C., Hunter, M. D., Pritikin, J. N., Zahery, M., Brick, T. R., . ., \& Boker, S. M. (2016). OpenMx 2.0: Extended structural equation and statistical modeling. Psychometrika, 81, 535-549. https//doi.org/10.1007/s11336-014-9435-8

Reeve, C. L. \& Charles, J. E. (2008). Survey of opinions on the primacy of $g$ and the social consequences of ability testing: A comparison of expert and non-expert views. Intelligence, 36, 681-688. https://doi.org/10.1016/j.intell.2008.03.007

R Core Team (2013). R: A language and environment for statistical computing. R Foundation for Statistical Computing, Vienna, Austria. URL http://www.R-project.org/.

Rhemtulla, M., van Bork, R., Epskamp, S., \& Isvoranu, A. M. (2018, May). In M. Rhemtulla (Chair), Network models and factor models: A theoretical, statistical, and clinical comparison. Symposium conducted at the $30^{\text {th }}$ annual meeting of the Association for Psychological Science, San Francisco, CA.

Rosseel, Y. (2012). lavaan: An R package for structrual equation modeling. Journal of Statistical Software, 48, 1-36. Retrieved from http://www.jstatsoft.org/v48/i02/

Sattler, J. M., \& Ryan, J. J. (2009). Assessment with the WAIS-IV. San Diego, CA: Jerome M. Sattler Publisher, Inc.

Schreiber, J. B., Nora, A., Stage, F. K., Barlow, E. A., \& King, J. (2006). Reporting structural equation modeling and confirmatory factor analysis results: A review. The Journal of Educational Research, 6, 323-338. https://doi.org/10.3200/JOER.99.6.323-338 
van Borkulo, C., Boschloo, L,. Borsboom, D., Penninx, B. W. J. H., Waldorp, L. J., Schoevers, R. A. (2015). Association of symptom network structure with the course of depression. JAMA Psychiatry, 72, 1219-1226. https://doi.org/10.1001/jamapsychiatry.2015.2079 van Buuren, S., \& Groothuis-Oudshoorn, K. (2011). mice: Multivariate imputation by chained equations in R. Journal of Statistical Software, 45, 1-67. https://www.jstatsoft.org/v45/i03/. van der Maas, H. L. J., Dolan, C. V., Grasman, R. P., Wicherts, J. M., Huizenga, H. M., \& Raijmakers, M. E. (2006). A dynamical model of general intelligence: The positive manifold of intelligence by mutualism. Psychological Review, 113, 842-861. https://doiorg.ccl.idm.oclc.org/10.1037/0033-295X.113.4.842

van der Maas, H. L. J., Kan, K.-J., Marsman, M., \& Stevenson, C. E. (2017). Network models for cognitive development and intelligence. Journal of Intelligence, 5, 16. https://doi.org/10.3390/jintelligence5020016 von Oertzen, T., Brandmaier, A.M., Tsang, S. (2015). Structural equation modeling with $\Omega$ nyx. Structural Equation Modeling: A Multidisciplinary Journal. doi:10.1080/10705511.2014.935842

Wechsler, D. (2008). Wechsler Adult Intelligence Scale Fourth Edition (WAIS-IV). Yong, A. G. \& Pearce, S. (2013). A beginner's guide to factor analysis: Focusing on exploratory factor analysis. Tutorials in Quantitative Methods for Psychology, 9, 79-94. doi: 10.20982/tqmp.09.2.p079 


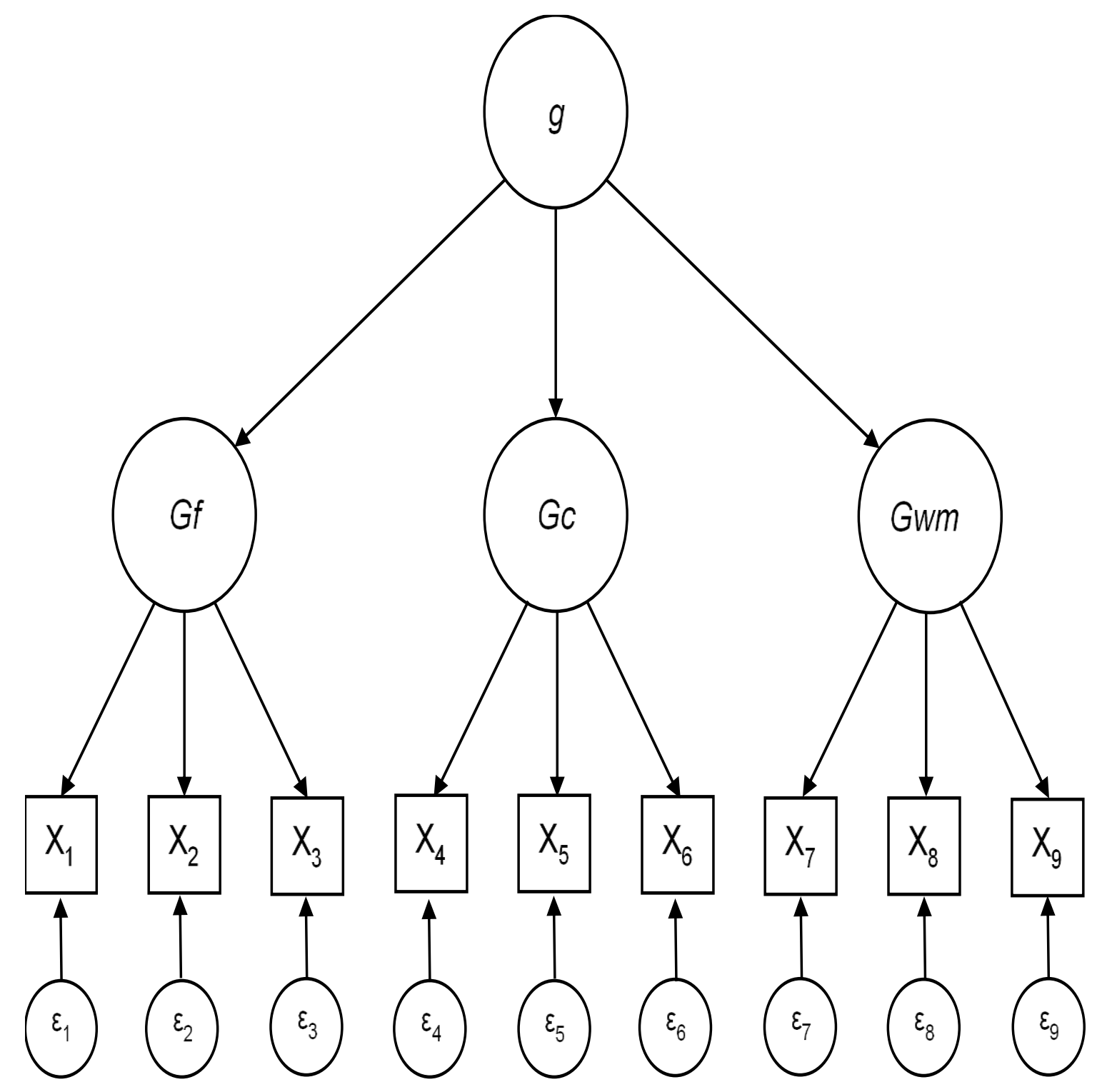

Figure 1. Example latent variable model: Higher-order model of intelligence based on CattellHorn-Carroll higher-order model of general intelligence. Adapted from "Human cognitive abilities: A survey of factor-analytic studies" by Carroll, 1993. Circles represent latent variables representing: general cognitive ability $(g)$, fluid intelligence $\left(G_{\mathrm{f}}\right)$, crystallized intelligence $\left(G_{\mathrm{c}}\right)$, and working memory $\left(G_{\mathrm{wm}}\right)$. Smaller circles presented at the bottom of the display represent measurement error $\left(\varepsilon_{i}\right)$ or random noise not explainable by latent variables. Should be noted that this is an abridged visualization of the Cattell-Horn-Carroll higher-order model of general intelligence. 
Table 1

Correlation Matrix and Descriptive Statistics of Hungarian Wechsler Adult Intelligence

Scale Fourth Edition

\begin{tabular}{|c|c|c|c|c|c|c|c|c|c|c|c|c|c|c|c|}
\hline & I & V & C & S & PC & $\mathrm{BD}$ & FW & MR & VP & A & DS & $\mathrm{LN}$ & $\mathrm{Ca}$ & $\mathrm{Cd}$ & SS \\
\hline I & 1.00 & .75 & .71 & .72 & .51 & .55 & .59 & .58 & .53 & .60 & .54 & .46 & .34 & .49 & .39 \\
\hline $\mathrm{V}$ & & 1.00 & .73 & .73 & .44 & .49 & .56 & .55 & .49 & .52 & .51 & .41 & .31 & .46 & .39 \\
\hline C & & & 1.00 & .72 & .44 & .51 & .57 & .55 & .48 & .52 & .47 & .40 & .30 & .44 & .36 \\
\hline $\mathrm{S}$ & & & & 1.00 & .50 & .57 & .60 & .55 & .54 & .55 & .52 & .45 & .34 & .48 & .38 \\
\hline PC & & & & & 1.00 & .55 & .57 & .50 & .56 & .52 & .45 & .38 & .36 & .43 & .41 \\
\hline $\mathrm{BD}$ & & & & & & 1.00 & .63 & .62 & .70 & .58 & .48 & .43 & .41 & .49 & .48 \\
\hline FW & & & & & & & 1.00 & .64 & .68 & .66 & .51 & .44 & .35 & .47 & .45 \\
\hline MR & & & & & & & & 1.00 & .61 & .57 & .51 & .41 & .35 & .50 & .44 \\
\hline VP & & & & & & & & & 1.00 & .59 & .49 & .41 & .39 & .44 & .45 \\
\hline A & & & & & & & & & & 1.00 & .62 & .52 & .37 & .42 & .42 \\
\hline DS & & & & & & & & & & & 1.00 & .57 & .34 & .49 & .45 \\
\hline LN & & & & & & & & & & & & 1.00 & .31 & .44 & .40 \\
\hline $\mathrm{Ca}$ & & & & & & & & & & & & & 1.00 & .49 & .54 \\
\hline $\mathrm{Cd}$ & & & & & & & & & & & & & & 1.00 & .67 \\
\hline SS & & & & & & & & & & & & & & & 1.00 \\
\hline$M$ & 9.99 & 9.99 & 10.00 & 9.98 & 10.03 & 9.99 & 9.99 & 10.00 & 9.99 & 9.99 & 10.00 & 9.30 & 10.05 & 9.99 & 9.98 \\
\hline$S D$ & 2.94 & 2.98 & 3.00 & 2.99 & 2.97 & 3.00 & 2.97 & 2.99 & 2.98 & 3.02 & 2.98 & 3.45 & 2.98 & 2.94 & 2.99 \\
\hline
\end{tabular}


Table 2

Model Fit Indices for Latent Variable and Network Models of Hungarian Wechsler Adult Intelligence Scale-Fourth Edition Data

\begin{tabular}{|c|c|c|c|c|c|c|c|}
\hline & \multicolumn{7}{|c|}{ Statistics } \\
\hline & Models & $\chi^{2}$ & $d f$ & CFI (TLI) & RMSEA & AIC & $\mathrm{BIC}^{2}$ \\
\hline \multirow{2}{*}{$\begin{array}{l}\text { lavaan/ } \\
\text { qgraph }\end{array}$} & WAIS-IV CFA & $376.44 * * *$ & 85 & $.97(.97)$ & .06 & 528.99 & 529.91 \\
\hline & WAIS-IV Network & 48.56 & 48 & $1.00(1.00)$ & .003 & 255.38 & 256.30 \\
\hline \multirow{2}{*}{ openMx } & WAIS-IV CFA & $376.43 * * *$ & 85 & $.97(.97)$ & .06 & 446.43 & 510.75 \\
\hline & WAIS-IV Network & $87.32 *$ & 60 & $1.00(1.00)$ & .02 & 207.32 & 317.58 \\
\hline \multicolumn{8}{|c|}{$\begin{array}{l}\text { Note. } * * * p<.001 ; * p<.05 ; \chi^{2}=\text { Model chi-square value; } d f=\text { degrees of freedom; AIC = Fit of } \\
\text { Akaike information criteria; BIC = Fit of sample size adjusted Bayesian information criteria; RMSEA } \\
=\text { Root mean square error of approximation; CFI = Comparative fit index; TLI = Tucker-Lewis index. } \\
\text { It should be noted that the difference in degrees of freedom between the two estimated network models } \\
\text { can be explained by the directed nature of the estimation technique used in the openMx package. }\end{array}$} \\
\hline
\end{tabular}

2 To compare AIC and BIC values across packages qgraph AIC and BIC values were transformed by dividing each by a product of two and the number of estimated parameters. 


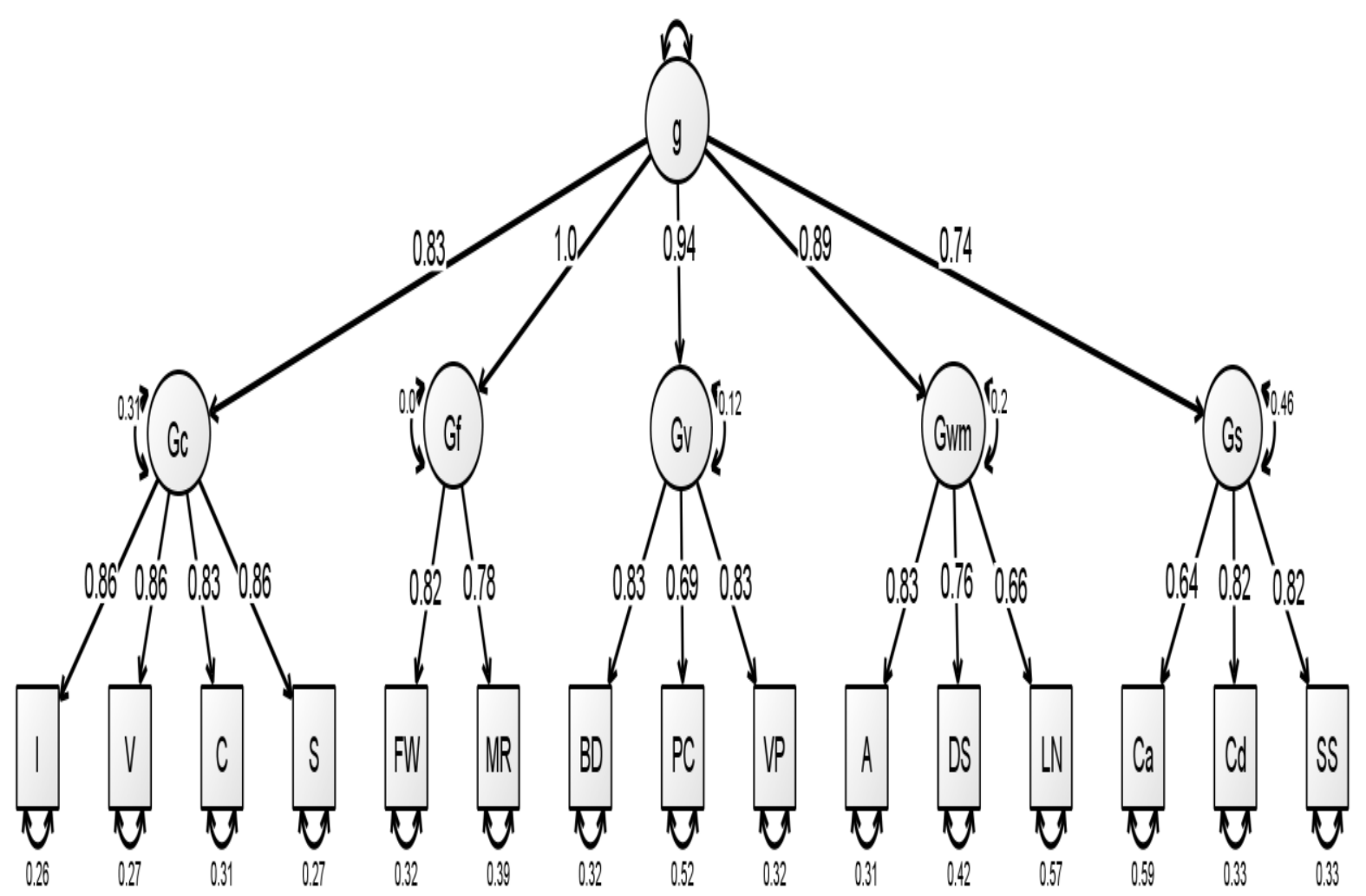

Figure 2. Hungarian Wechsler Adult Intelligence Scale Fourth Edition data applied to the Cattell-Horn-Carroll higher-order model of intelligence. All values are standardized values from the confirmatory factor analysis conducted using lavaan. Figure generated using Onyx. 


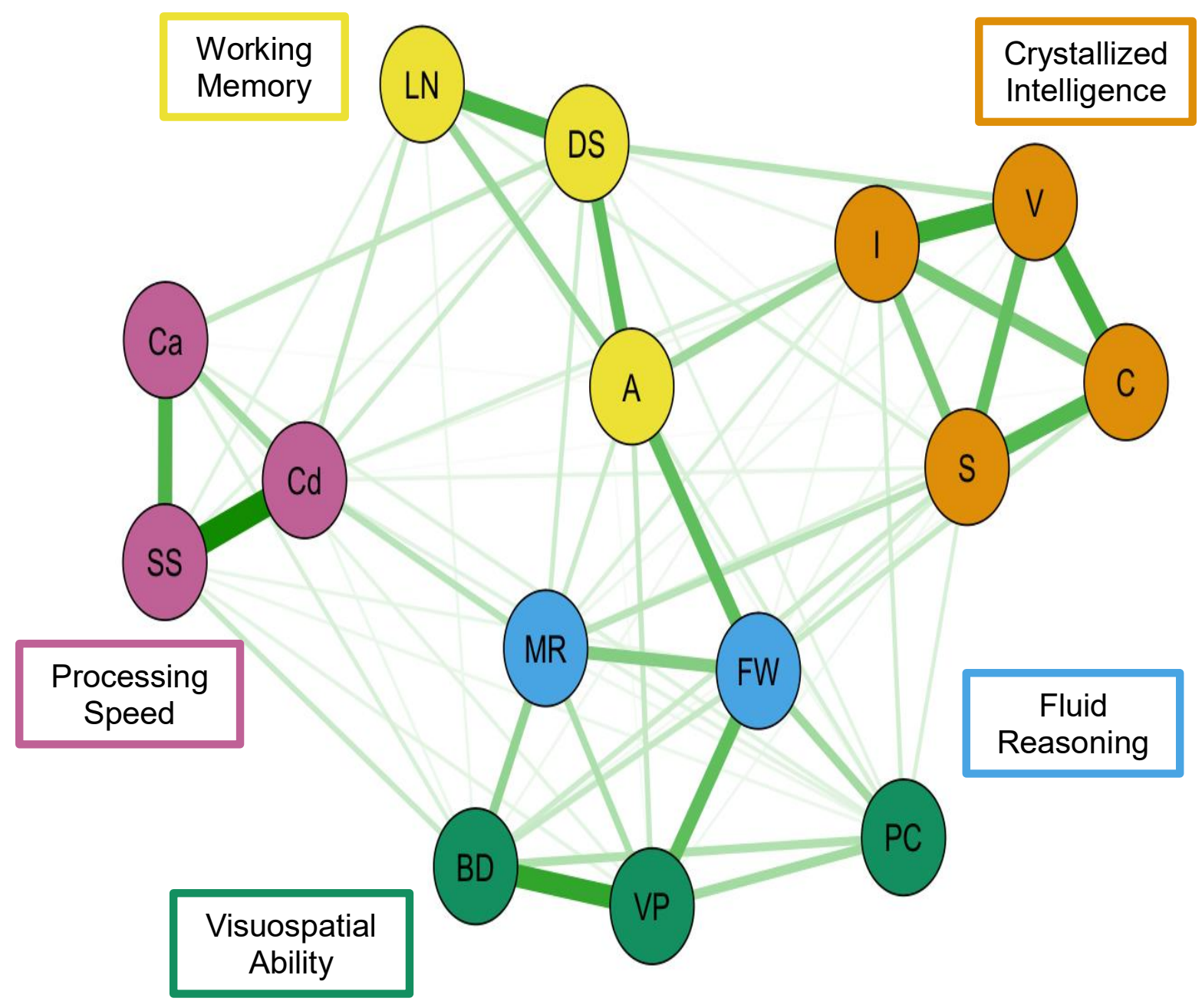

Figure 3. Hungarian Wechsler Adult Intelligence Scale Fourth Edition psychometric network model estimated using qgraph. Nodes are colored to represent the underlying latent factor as theorized by the Cattell-Horn-Carroll higher-order model of intelligence. Green edges indicate positive partial correlations between nodes. 


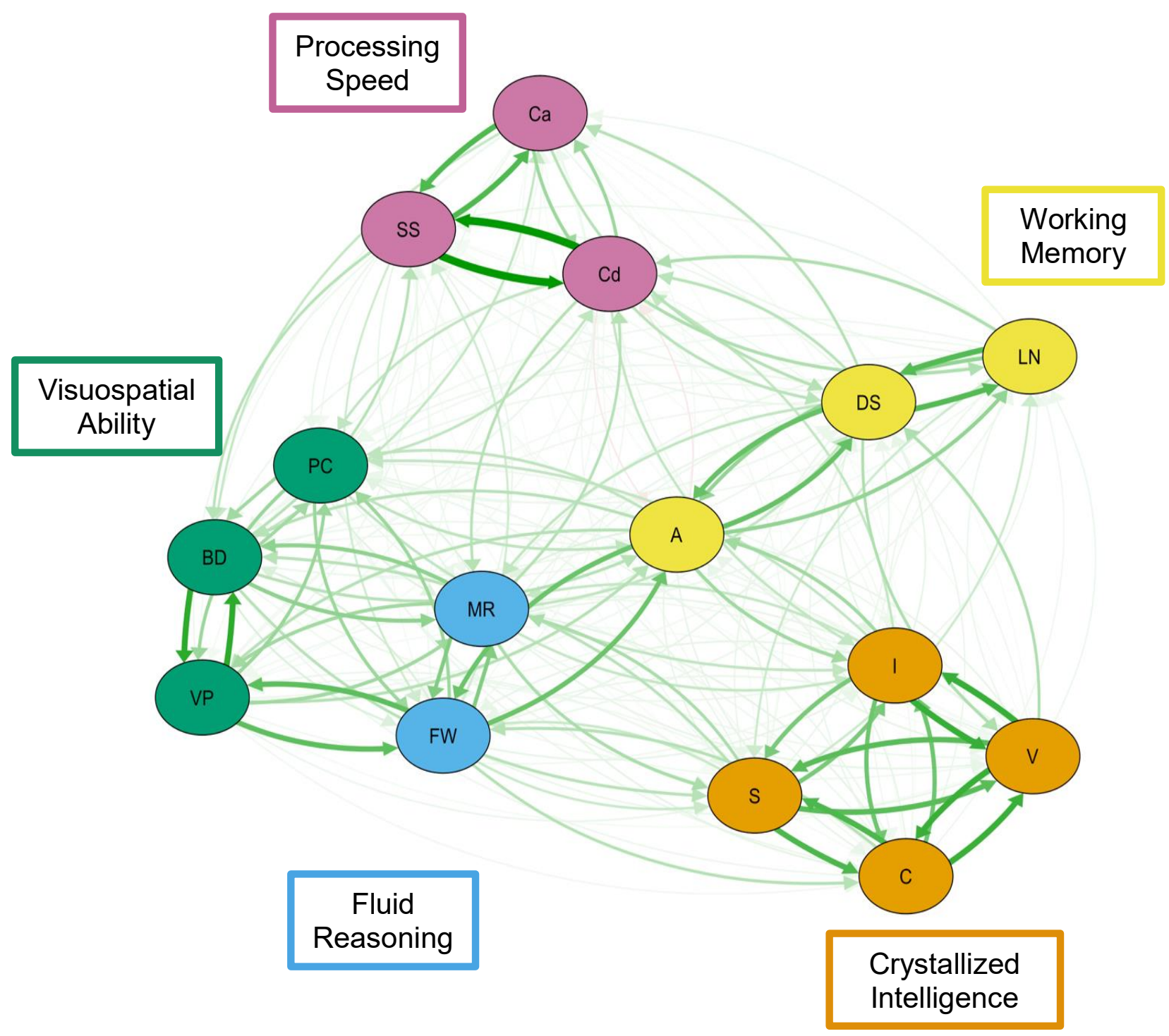

Figure 4. Hungarian Wechsler Adult Intelligence Scale Fourth Edition psychometric network model estimated using openMx. Nodes are colored to represent the underlying latent factor as theorized by the Cattell-Horn-Carroll higher-order model of intelligence. Green edges indicate positive partial correlations and red edges indicate negative partial correlations between nodes. 